\title{
NP Animacy Identification for Anaphora Resolution
}

\author{
Constantin Orăsan \\ Richard Evans \\ Research Group in Computational Linguistics \\ School of Humanities, Languages and Social Sciences \\ University of Wolverhampton \\ Stafford St., Wolverhampton, WV1 $1 S B$ \\ United Kingdom
}

\author{
C.Orasan@WLV.AC.uK \\ R.J.EVANS@WLV.AC.UK
}

\begin{abstract}
In anaphora resolution for English, animacy identification can play an integral role in the application of agreement restrictions between pronouns and candidates, and as a result, can improve the accuracy of anaphora resolution systems. In this paper, two methods for animacy identification are proposed and evaluated using intrinsic and extrinsic measures. The first method is a rule-based one which uses information about the unique beginners in WordNet to classify NPs on the basis of their animacy. The second method relies on a machine learning algorithm which exploits a WordNet enriched with animacy information for each sense. The effect of word sense disambiguation on the two methods is also assessed. The intrinsic evaluation reveals that the machine learning method reaches human levels of performance. The extrinsic evaluation demonstrates that animacy identification can be beneficial in anaphora resolution, especially in the cases where animate entities are identified with high precision.
\end{abstract}

\section{Introduction}

Anaphora resolution is the process which attempts to determine the meaning of expressions such as pronouns or definite descriptions whose interpretation depends on previously mentioned entities or discourse segments. Anaphora resolution is very important in many fields of computational linguistics such as machine translation, natural language understanding, information extraction and text generation (Mitkov, 2002).

Previous work in anaphora resolution (AR) has shown that its levels of performance are related to both the type of text being processed and to the average number of noun phrases (NPs) under consideration as a pronoun's antecedent (Evans \& Orăsan, 2000). Acknowledging this, researchers have proposed and incorporated various methods intended to reduce the number of candidate NPs considered by their anaphora resolution systems. Most approaches to pronominal anaphora resolution rely on compatibility of the agreement features between pronouns and antecedents, as a means of minimising the number of NP candidates. Although, as noted by Barlow (1998) and Barbu, Evans, and Mitkov (2002), this assumption does not always hold, it is reliable in enough cases to be of great practical value in anaphora and coreference resolution systems. Such systems rely on knowledge about the number and gender of NP candidates in order to check the compatibility between pronouns and candidates (Hobbs, 1976; Lappin \& Leass, 1994; Kennedy \& Boguraev, 1996; Mitkov, 1998; Cardie \& Wagstaff, 1999; Ng \& Cardie, 2002). In addition to number and 
gender compatibility, researchers reduced the number of competing candidates considered by their systems by means of syntactic filters (Hobbs, 1976; Lappin \& Leass, 1994), semantic filters (Hobbs, 1978) or discourse structure (Brennan, Friedman, \& Pollard, 1987; Cristea, Ide, Marcu, \& Tablan, 2000).

In English, the automatic identification of the specific gender of NPs is a difficult task of arguably limited utility. Despite this, numerous researchers (Hale \& Charniak, 1998; Denber, 1998; Cardie \& Wagstaff, 1999) have proposed automatic methods for identifying the potential gender of NPs' referents. In this paper, the problem of animacy identification is tackled. The concern with animacy as opposed to gender arises from the observation that animacy serves as a more reliable basis for agreement between pronouns and candidates (see examples in Section 2). Animacy identification can be very useful in tasks like anaphora resolution and coreference resolution where the level of ambiguity can be reduced by filtering out candidates which do not have the same value for animacy as the anaphor, as well as in question answering, where it can be used to improve system responses to "who" questions by allowing them to ensure that the generated answers consist of animate references.

In this research, a NP is considered to be animate if its referent can also be referred to using one of the pronouns he, she, him, her, his, hers, himself, herself, or a combination of such pronouns (e.g. his/her). Section 2 provides more clarity on this definition, considering a range of exceptions and problematic cases, as well as examining some consequences of this treatment of animacy. The corpus used in this research is described in Section 3. In this paper several methods for animacy identification are proposed and evaluated. First, a simple statistical method based on WordNet (Fellbaum, 1998) is described in Section 4.1. Following from the description of the simple statistical method, a machine learning method that overcomes some of the problems of the simple method, offering improved performance, is described in Section 4.2. In the latest stages of development, word sense disambiguation (WSD) is added to further improve the accuracy of the classification. This is presented in Section 4.3. In Section 5, the systems are evaluated using both intrinsic and extrinsic evaluation methods, and it is noted that the machine learning methods reach human performance levels. Finally, Section 6 is dedicated to related work and is followed by conclusions.

\section{What Constitutes an Animate Noun Phrase?}

It has been argued that "in English nouns are not classified grammatically, but semantically according to their coreferential relations with personal, reflexive and wh-pronouns" (Quirk, Greenbaum, Leech, \& Svartvik, 1985, p. 314). According to their classification, animate noun phrases contain both personal (e.g. male, female, dual, common and collective nouns) and non-personal noun phrases (e.g. common, collective and animal nouns). In this paper, our goal is to design a method which improves the performance of anaphora resolution methods by filtering out candidates which do not agree in terms of animacy with a given referential pronoun. For this reason, the more specific definition of animacy given in the introduction is used. This means that in this paper, those noun phrases which can normally be referred to by the pronouns he and she and their possessive and reflexive forms, are considered animate, but no distinction is made between those pronouns to determine their gender. This view is adopted because, in the linguistic processing of English documents, it 


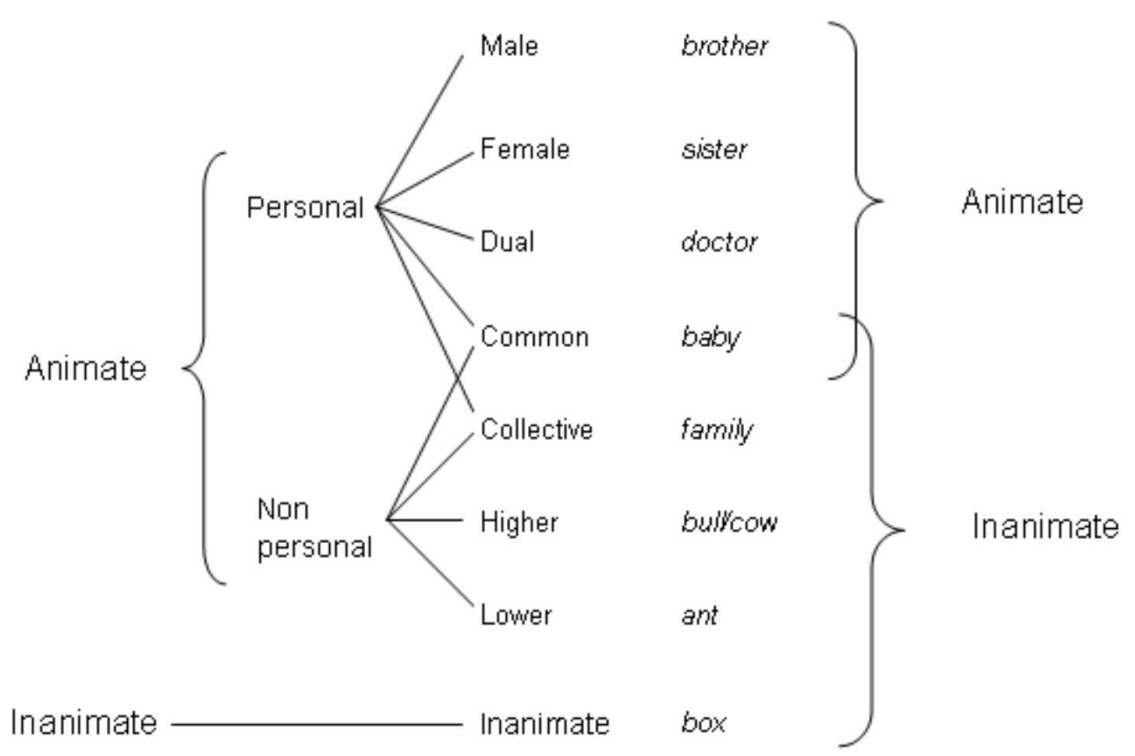

Figure 1: Quirk el. al. (1985) vs our classification of animacy. (Adapted from Quirk el. al. (1985, p. 314, Fig. 5.104))

is vital to distinguish between neuter and animate references but problematic, and often of limited utility, to distinguish between masculine and feminine ones.

To illustrate, in the sentence The primary user of the machine should select his or her own settings, considering the noun phrase the primary user of the machine to be either masculine or feminine, and then applying strict agreement constraints between this reference and the subsequent pronominal ones, will adversely affect the performance of reference resolution systems because such constraints will eliminate the antecedent from the list of candidates of one of the pronouns depending on the gender attached to the NP. Ideally, the reference should be considered animate - compatible, in terms of agreement, with subsequent animate pronouns, and incompatible with neuter pronouns.

Figure 1 presents the differences between Quirk et. al.'s (1985) classification of animacy and the one used in this paper. As can be seen in the figure, their definition of animate nouns is much wider than that used in this paper. We consider of the classes presented by Quirk et al.'s (1985), the only animate entities to be male, female, dual ${ }^{1}$ and some common gender ${ }^{2}$ nouns.

Common gender nouns are defined by Quirk et al. (1985) as intermediate between personal and non-personal nouns. For us, their animacy can be either animate or inanimate, depending on which pronoun is used to refer to them. However, the animacy of some of the common nouns such as cat depends upon the perception of that entity by the speaker/writer. If the noun is being used to refer to a pet, the speaker/writer is also likely to use animate

1. Dual nouns are nouns that refer to people but whose gender is underspecified such as artist, cook, etc.

2. In Figure 1 these nouns are labeled as common. 
pronouns rather than inanimate ones to refer to it. Such circumstances are not detected by our method: they may be the focus of methods which try to identify the sentiments of speakers/writers towards entities.

Collective nouns such as team, that refer to sets of animate entities, may intuitively be considered animate. However, the only suitable pronominal references for the denotation of such phrases are singular neuter pronouns or plural pronouns of unspecified gender. These referents are never referred to using animate pronouns. Given that the raison d'etre for our research into animacy identification is the facilitation of real-world anaphora resolution, such NPs are considered inanimate in the current work, and not animate as they are by Quirk et al. (1985).

Collective nouns such as people pose further problems to annotation and processing. In some contexts the word people can be used as the plural form of person, in which case it should be considered animate by the definition presented earlier. However in some cases it is used more generically to refer to national populations (e.g. the peoples of Asia) in which case it should be considered inanimate. In light of this, it seems that the class of this word depends on its context. However, in practical terms, the morpho-syntactic parsing software that we use (Tapanainen \& Järvinen, 1997) returns people and not person as the root the noun, so for this reason, the noun people is considered inanimate for our purposes. The same reasoning was applied to other similar nouns. The drawback of this approach is that annotators did not find this very intuitive and as a result errors were introduced in the annotation (as discussed in the next section).

The rest of the categories introduced by Quirk et al. (1985): non-personal higher, nonpersonal lower and inanimate correspond in our definition to inanimate nouns. As with common gender nouns, it is possible to have non-personal higher and lower nouns such as horse and rabbit which can be pets and therefore are referred to by speakers using he or she. As we cannot detect such usages, they are all considered inanimate. ${ }^{3}$

In the present work, the animacy of a noun phrase (NP) is considered to derive from the animacy of its head. To illustrate, both the man and the dead man can be referred to using the same animate pronoun. Moreover, when considering the animacy of plural NPs such as mileage claimants, the singular form mileage claimant is derived and used as the basis of classification because the plural form shares the animacy of its singular form. In this way, our treatment of NP animacy mirrors the treatment of grammatical number under the Government and Binding Theory (Chomsky, 1981). Under this approach, the projection principle implies that agreement information for a NP is derived from that of its head.

In this paper, the animacy of only common nouns is determined and not of proper nouns such as named entities (NE). The reason for this is that the separate task of named entity recognition is normally used to classify NEs into different categories such as PERSON, ORGANizATION, and LOCATION. Given that they label entities of similar semantic types, these categories can then be used to determine the animacy of all the entities that belong to them. It is acknowledged that named entity recognition is an important component in the identification of animate references, but one which lies beyond the scope of the present work. Methods based on semantics, such as the ones described in Section 4 are especially vulnerable to errors caused by a failure to recognise the difference between words such as

3. Actually on the basis of the explanation provided by Quirk et al. (1985) the distinction between common nouns and higher and lower non-personal nouns when the latter are 'personified' seems very fuzzy. 


\begin{tabular}{|l|c|c|}
\hline & SEMCOR & AI \\
\hline No of words & 104,612 & 15,767 \\
\hline No of animate entities & 2,321 & 538 \\
\hline No of inanimate entities & 17,380 & 2,586 \\
\hline Percentage of animate entities & $12 \%$ & $21 \%$ \\
\hline Total entities & 19,701 & 3,124 \\
\hline
\end{tabular}

Table 1: The characteristics of the two corpora used

$C a t$ or $B o b$ when used as common nouns which are inanimate references or as proper nouns which are animate references.

\section{Corpus-Based Investigation}

The identification of NP animacy, as described in the previous section, was amenable to a corpus-based solution. In this research two corpora are being used: The first is a collection of texts from Amnesty International (AI) which were selected because they contain a relatively large proportion of references to animate entities. The second is a selection of texts from the SEMCOR corpus (Landes, Leacock, \& Tengi, 1998), chosen because their nouns were annotated with senses from WordNet. This annotation made them suitable for exploitation in the development of the automatic method for animacy identification described in Section 4.2. The SEMCOR corpus was built on the basis of Brown Corpus (Frances \& Kucera, 1982) and for our experiments we use texts from newswire, science, fiction and humor.

In order to make the data suitable for evaluation purposes, NPs from the two corpora have been manually annotated with information about their animacy. The characteristics of these corpora are summarized in Table 1. As can be seen in the table, even though texts which contain many references to animate entities were selected, the number of inanimate entities is still much larger than the number of animate ones.

To assess the difficulty of the annotation task, and implicitly, to estimate the upper performance limit of automatic methods, a second annotator was asked to annotate a part of the corpus and inter-annotator agreement was calculated. To this end, the whole AI corpus, and nine texts with over 3,500 references from the SEMCOR corpus have been randomly selected and annotated. Comparison between the two annotations revealed a level of agreement of $97.5 \%$ between the two annotators and a value of 0.91 for the kappa statistic which indicates very high agreement between annotators. The agreement on the SEMCOR data was slightly higher than that for the AI corpus, but the difference was not statistically significant.

Investigation of the annotation performed by the two annotators and discussion with them revealed that the main source of disagreement was the monotony of the task. The two annotators had to use a simple interface which displayed for each sentence one NP at a time, and were required to indicate whether the NP was animate or inanimate by choosing one of two key strokes. Due to the large number of inanimate entities in the corpus, the annotators often marked animate entities as inanimate accidentally. In some cases they noticed their mistake and corrected it, but it is very likely that many such mistakes went unobserved. 
Another source of disagreement were collective nouns such as people, government, jury or folk which according to the discussion in Section 2 should normally be marked as inanimate. In some cases, the context of the NP or tiredness on the part of the annotator led to them being erroneously marked as animate. Similarly, it was noticed that the annotators wrongly considered some plural noun phrases such as observers, delegates, communists, and assistants to be collective ones and marked them as inanimate. However, it is likely that some of these errors were introduced due to the monotony of the task. Unfamiliar nouns such as thuggee, and words used in some specialized domains such as baseball also caused difficulties. Finally, another source of error arose from the use of Connexor's FDG Parser (Tapanainen \& Järvinen, 1997) to identify the noun phrases for annotation. As a result, some of the noun phrases recognized by the system were ambiguous (e.g. specialists and busy people was presented as one NP and according to the definition of animacy adopted in the present work, specialists is animate, whereas busy people is inanimate ${ }^{4}$.).

\section{Methods for Animacy Identification}

By contrast to the situation with proper name recognition and classification, which can exploit surface textual clues such as capitalization and the explicit occurrence of words in a small gazetteer of titles, knowledge as to the animacy of common NPs appears to be purely implicit. Recognition of references to animate entities must, at some point, be grounded in world-knowledge and computed from explicit features of the text. This section presents two methods developed for animacy identification which rely on information extracted from WordNet, an electronic lexical resource organized hierarchically by relations between sets of synonyms or near-synonyms called synsets (Fellbaum, 1998). The first method is a rulebased one which employs a limited number of resources and is presented in Section 4.1. Its shortcomings are addressed by the machine learning method presented in Section 4.2. Both methods consider all the senses of a word before taking a decision about its animacy. For this reason, the word sense disambiguation (WSD) module briefly discussed in Section 4.3 was integrated into them.

\subsection{Rule-Based Method}

In WordNet, each of the four primary classes of content-words (nouns, verbs, adjectives and adverbs) are arranged under a small set of top-level hypernyms called unique beginners (Fellbaum, 1998). Investigation of these unique beginners revealed that several of them were of interest with respect to the aim of identifying the animate entities in a text. In the case of nouns there are 25 unique beginners, three of which are expected to be hypernyms of senses of nouns that usually refer to animate entities. These are animal, reference number (05), person (18), and relation (24). ${ }^{5}$ There are also four verb sense hierarchies out of fourteen, that allow the inference to be made that their subject NPs should be animate. The unique beginners in these cases are cognition (31), communication (32), emotion (37) and social

4. It can be argued that the singular form of people is person, and that it should therefore be marked as animate. However, as discussed in Section 2 due to the way it is processed by the preprocessing tools employed here, annotators were asked to consider it inanimate

5. The unique beginner animal corresponds to both animate and inanimate entities while relation subsumes mainly human relationships such as brother, sister, parent, etc. 
(41). ${ }^{6}$ It has been noted that inanimate entities such as organizations and animals can also be agents of these types of verb, but it is expected in the general case that these instances will be rare enough to ignore. In light of the way in which WordNet is organized, it was clear that it could be exploited in order to associate the heads of noun phrases with a measure of confidence that the associated NP has either an animate or inanimate referent.

It is very common for a noun to have more than one meaning, in many cases corresponding to sense hierarchies which start from different unique beginners. For this reason, the decision about whether a noun phrase is animate or inanimate should be taken only after all the possible senses of the head noun have been consulted. Given that some of these senses are animate whilst others are inanimate, an algorithm which counts the number of animate senses that are listed for a noun (hyponyms of unique beginners 05, 18, or 24) and the number of inanimate senses (hyponyms of the remaining unique beginners) was proposed. Two ratios are then computed for each noun:

$$
\begin{gathered}
\text { Noun animacy }(N A)=\frac{\text { Number of animate senses }}{\text { Total number of senses }} \\
\text { Noun inanimacy }(N I)=\frac{\text { Number of inanimate senses }}{\text { Total number of senses }}
\end{gathered}
$$

and compared to pre-defined thresholds in order to classify them as animate or inanimate. Similarly, in the case of nouns that are the heads of subject NPs, counts are made of the animate and inanimate senses of the verbs they are subjects of and used to calculate Verb animacy (VA) and Verb inanimacy (VI) in the same way as NA and NI. These ratios are also used to determine the animacy of the subject NP. Finally, contextual rules (e.g. the presence of NP-internal complementizers and reflexives such as who or herself) are applied in order to improve the classification. The algorithm is presented in Algorithm 1 and evaluated in Section 5. The three thresholds used in the algorithm were determined through experimentation and the best values were found to be $t_{1}=0.71, t_{2}=0.92$ and $t_{3}=0.90$.

\subsection{Machine Learning for Animacy Identification}

The method presented in the previous section has two main weaknesses. The first one is that the unique beginners used to determine the number of animate/inanimate senses are too general, and in most cases they do not reliably indicate the animacy of each sense in the class. The second weakness is due to the naïve nature of the rules that decide whether a NP is animate or not. Their application is simple and involves a comparison of values obtained for a NP with threshold values that were determined on the basis of a relatively small number of experiments. In light of these problems, a two step approach, each addressing one of the aforementioned weaknesses, was proposed. In the first step, an annotated corpus is used to determine the animacy of WordNet synsets. This process is presented in Section 4.2.1. Once this information is propagated through the whole of WordNet, it is used by a machine learning algorithm to determine the animacy of NPs. This method is presented in Section 4.2.2.

6. The social unique beginner subsumes relations such as abdicate, educate and socialize. 


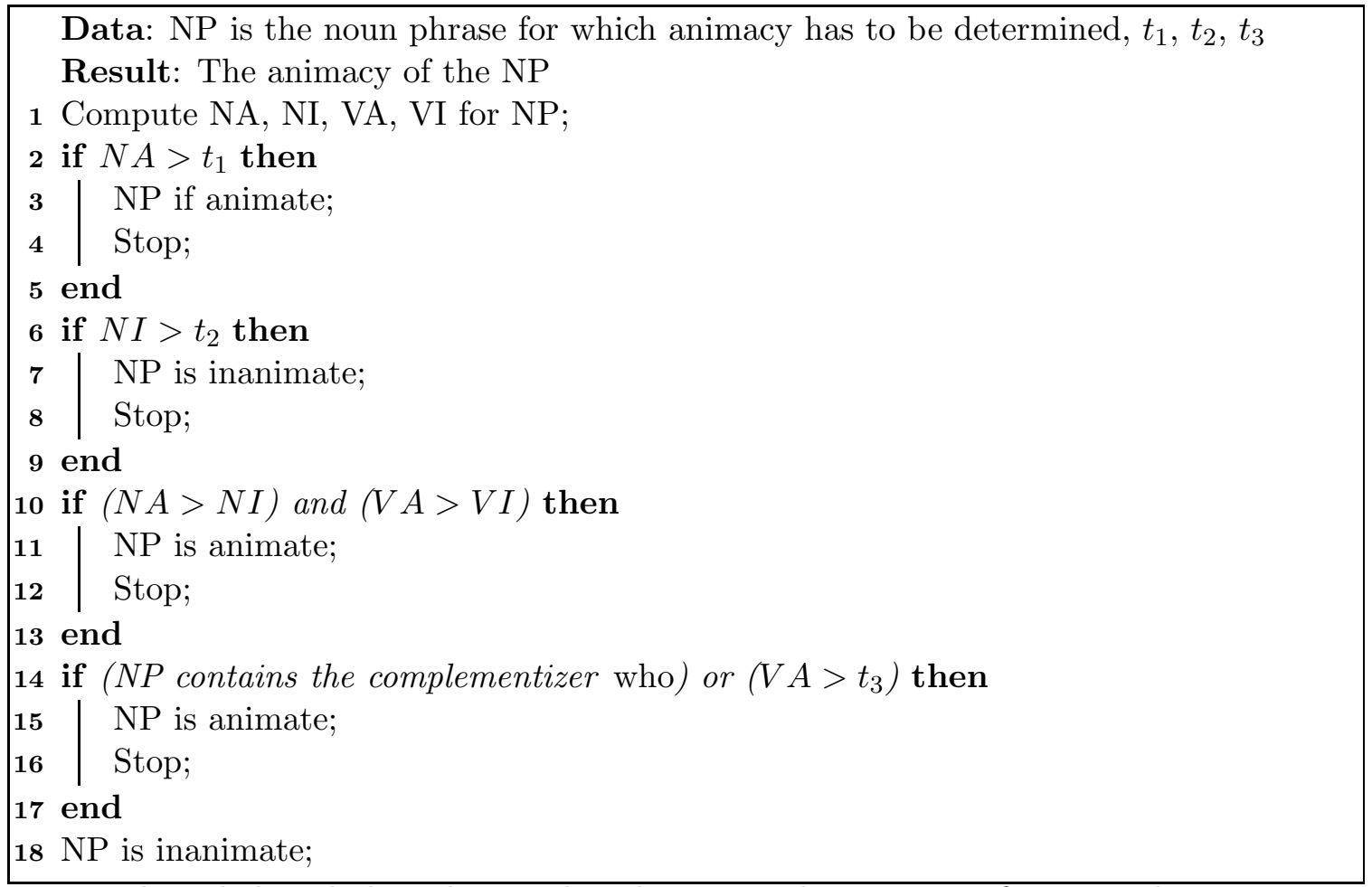

1: The rule-based algorithm used to determine the animacy of a noun phrase

\subsubsection{The Classification of the Senses}

As previously mentioned, the unique beginners are too general to be satisfactorily classified as wholly animate or inanimate. However, this does not mean that it is not possible to uniquely classify more specific senses as animate or inanimate. In this section, a corpusbased method which classifies synsets from WordNet according to their animacy is presented.

The starting point for classifying the synsets was the information present in our annotated version of the SEMCOR corpus. The reason for this is that by adding our animacy annotation to nouns which were annotated with their corresponding sense from WordNet, this information could be used to determine the animacy of the synset. However, due to linguistic ambiguities and tagging errors not all the senses can be classified adequately in this way. Moreover, many senses from WordNet do not appear in SEMCOR, which means that no direct animacy information can be determined for them. In order to address this problem, the decision was made to use a bottom up procedure which begins by classifying unambiguous terminal nodes and then propagates this information to more general nodes. A terminal node is unambiguously classified using the information from the annotated files if all its occurrences in the corpus are annotated with the same class. In the same way, a more general node can be unambiguously classified if all of its hyponyms have been assigned to the same class.

Due to annotation errors or rare uses of a sense, this condition is rarely met and a statistical measure must be employed in order to test the animacy of a more general node. A simple approach which classifies a synset using a simple voting procedure on behalf of its 


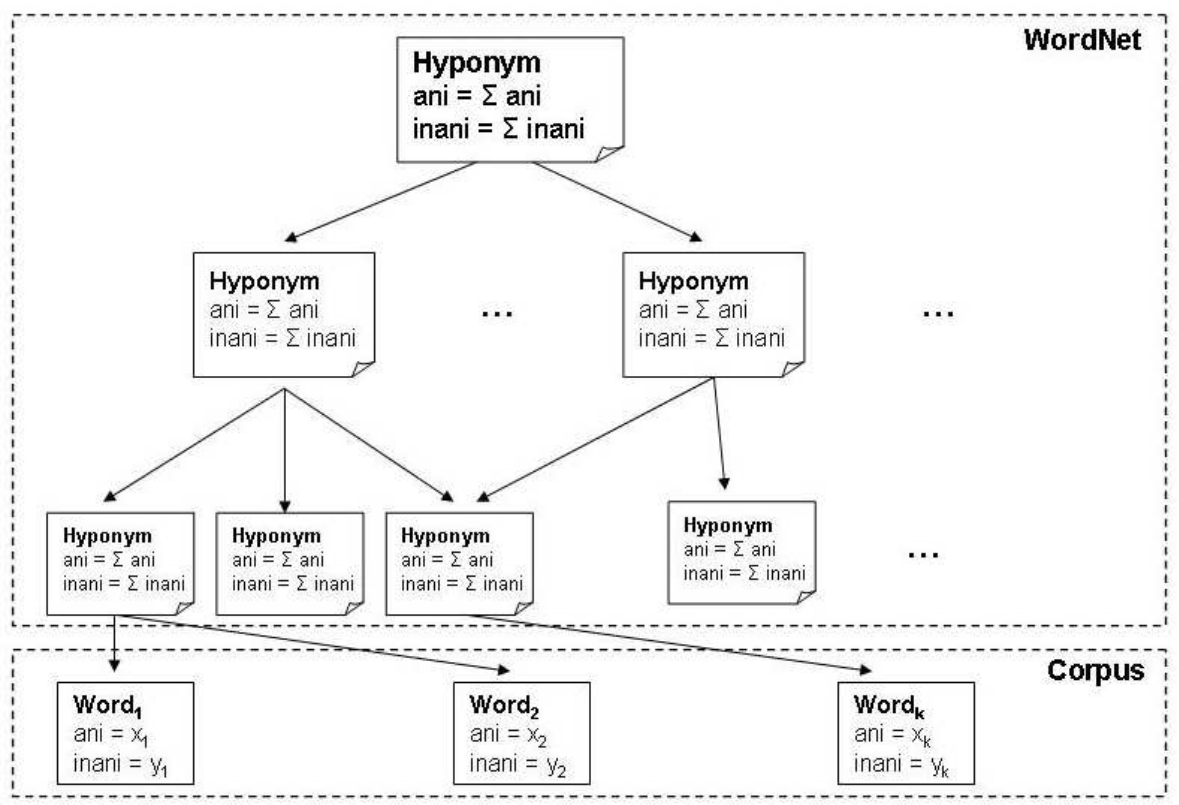

Figure 2: Example showing the propagation of animacy from the corpus to more general senses

hyponyms will be unsatisfactory because it is necessary to know when a node is too general to be able to assign it to one of the classes. For this reason a statistical measure was used to determine the animacy of a node in ambiguous cases.

The statistical measure used in this process is chi-squared, a non-parametric test which can be used to estimate whether or not there is any significant difference between two different populations. In order to test whether or not a node is animate, the two populations to be compared are:

1. an observed population which consists of the senses of the node's hyponyms which were annotated as animate, and

2. a hypothetical population in which all of the node's hyponyms are animate.

If chi-square indicates that there is no difference between the two populations then the node is classified as animate. The same process is repeated in order to classify an inanimate node. If neither test is passed, it means that the node is too general, and it and all of its hypernyms can equally refer to both animate and inanimate entities. In unambiguous cases (i.e. when all the hyponyms observed in the corpus ${ }^{7}$ are annotated as either animate or inanimate, but not both), the more general node is classified as its hyponyms are. The way in which information is propagated from the corpus into WordNet is presented in Figure 2.

To illustrate, for a more general node which has $n$ hyponyms the contingency table (Table 2) can be built and used to determine its animacy. Each hyponym is considered

7. Either directly or indirectly via hyponymy relations. 


\begin{tabular}{|c|c|c|c|c|c|}
\hline & Sense $_{1}$ & Sense $_{2}$ & Sense $_{3}$ & $\ldots$ & Sense $_{n}$ \\
\hline Observed & ani $_{1}$ & ani $_{2}$ & ani $_{3}$ & $\ldots$ & ani $_{n}$ \\
\hline Expected & ani $_{1}+$ inani $_{1}$ & ani $_{2}+$ inani $_{2}$ & ani $_{3}+$ inani $_{3}$ & $\ldots$ & ani $_{n}+$ inani $_{n}$ \\
\hline
\end{tabular}

Table 2: Contingency table for testing the animacy of a hypernym

to have two attributes: the number of times it has been annotated as animate $\left(a n i_{i}\right)$ and the number of times it has been annotated as inanimate $\left(\right.$ inani $\left._{i}\right)$. The figures for $a n i_{i}$ and inani $_{i}$ include both the number of times that the sense directly appears in the corpus and the number of times it appears indirectly via its hyponyms. Given that the system is testing to see whether the more general node is animate or not, for each of its hyponyms, the total number of occurrences of a sense in the annotated corpus is the expected value (meaning that all the instances should be animate and those which are not marked as animate are marked that way because of annotation error or rare usage of the sense) and the number of times the hyponym is annotated as referring to an animate entity is the observed value. Chi-square is calculated, and the result is compared with the critical level obtained for $n-1$ degrees of freedom and a significance level of .05. If the test is passed, the more general node is classified as animate.

In order to be a valid test of significance, chi-square usually requires expected frequencies to be 5 or more. If the contingency table is larger than two-by-two, some few exceptions are allowed as long as no expected frequency is less than one and no more than $20 \%$ of the expected frequencies are less than 5 (Sirkin, 1995). In the present case it is not possible for expected frequencies to be less than one because this would entail no presence in the corpus. If, when the test is applied, more than $20 \%$ of the senses have an expected frequency less than 5 , the two similar senses with the lowest frequency are merged and the test is repeated. ${ }^{8}$ If no senses can be merged and still more than $20 \%$ of the expected frequencies are less than 5 , the test is rejected.

This approach is used to classify all the nodes from WordNet as animate, inanimate or undecided. The same approach is also employed to classify the animacy of verbs on the basis of the animacy of their subjects. An assessment of the coverage provided by the method revealed that almost $94 \%$ of the nodes from WordNet can be classified as animate or inanimate. This is mainly due to the fact that some very general nodes such as person, plant or abstraction can be classified without ambiguity and as a result all their hyponyms can be classified in the same way. This enriched version of WordNet is then used to classify nouns as described in the next section.

\subsubsection{The Classification of a Noun}

The classification described in the previous section is useful for determining the animacy of a sense, even for those which were not previously found in the annotated corpus, but which are hyponyms of a node that has been classified. However, nouns whose sense is unknown cannot be classified directly and therefore an additional level of processing is necessary. In

8. In this context, two senses are considered similar if they both have the same attribute (i.e. animacy or inanimacy) equal to zero. 
this section, the use of Timbl (Daelemans, Zavrel, van der Sloot, \& van den Bosch, 2000) to determine the animacy of nouns is described.

TimBL is a program which implements several machine learning techniques. Experimenting with the algorithms available in TimBL with different configurations, the best results were obtained using instance-based learning with gain ratio as the weighting measure (Quinlan, 1993; Mitchell, 1997). In this type of learning, all the instances are stored without trying to infer anything from them. At the classification stage, the algorithm compares a previously unseen instance with all the data stored at the training stage. The most frequent class in the $\mathrm{k}$ nearest neighbors is assigned as the class to which that instance belongs. After experimentation, it was noticed that the best results were obtained when the three nearest neighbors were used $(k=3)$, the distance between two instances is calculated using overlap metric and the importance of each feature is weighted using gain ratio (Daelemans et al., 2000).

In the present case, the instances used in training and classification consist of the following information:

1. The lemma of the noun which is to be classified.

2. The number of animate and inanimate senses of the word. As mentioned before, in the cases where the animacy of a sense is not known, it is inferred from its hypernyms. If this information cannot be found for any of a word's hypernyms, information on the unique beginners for the word's sense is used, in a manner similar to that used by the rule-based system described in Section 4.1.

3. For the heads of subject NPs, the number of animate/inanimate senses of its verb. For those senses for which the classification is not known, an algorithm similar to the one described for nouns is employed. These values are 0 for heads of non-subjects.

4. The ratio of the number of animate singular pronouns (e.g he or she) to inanimate singular pronouns (e.g. it) in the whole text. The justification for this feature is that a text containing a large number of gender marked pronouns will be more likely to mention many animate entities

These features were encoded as vectors to be classified by TimBL using the algorithm and settings described earlier. The algorithm described in this section is evaluated in Section 5 .

\subsection{Word Sense Disambiguation}

It is difficult to disambiguate the possible senses of words in unrestricted texts, but it is not so difficult to identify those senses which are more likely to be used in a text than others. Such information was not considered in the methods presented in Sections 4.1 and 4.2. Instead, in those methods, all the senses were considered to have an equal weight. In order to address this shortcoming, the word sense disambiguation (WSD) method described by Resnik (1995) was implemented and used in the classification algorithm. The WSD method computes the weight of each possible sense of each noun by considering the other nouns in a text. These weights were used to compute the number of animate/inanimate senses. The underlying hypothesis is that the animacy/inanimacy of senses which are more likely to be 
used in a particular text should count more than that of improbable senses. The impact of this approach on the animacy identifiers presented in the previous section is also evaluated.

\section{Evaluation of the Systems}

In this section, the systems presented in Section 4 are evaluated using intrinsic and extrinsic evaluation methods (Sparck Jones \& Galliers, 1996). Both evaluation methods are necessary because the aim is not only to find out which of the methods can classify references to animate entities most accurately, but also to assess how appropriate they are for inclusion into an anaphora resolution method. In addition, the complexity of the systems is considered.

In order to increase the reliability of the evaluation, the systems are assessed on both corpora described in Section 3. The thresholds used in the simple method presented in Section 4.1 were determined through direct observation of the performance results when the system was applied to the AI corpus. Evaluating the method on the SEMCOR corpus allows its performance to be measured on completely unseen data. In addition, the texts from SEMCOR are in a completely different genre from AI, allowing an assessment to be made of the degree to which the system described in Section 4.1 is genre independent.

Evaluation raises more serious problems when the machine learning method is considered. As is well known, whenever a machine learning method is evaluated, a clear distinction has to be made between training data and testing data. In the case of the system described in Section 4.2, the approach was evaluated using 10-fold cross-validation over the SEMCOR corpus. Given that the AI corpus is available, the systems can also be evaluated on data from a domain which was not used in setting the parameters of the machine learning method. In addition, the evaluation of the machine learning methods on the AI corpus is useful in proving that the classification of the synsets from WordNet on the basis of the animacy annotation added to SEMCOR can be used to develop a system whose performance is not text-dependent.

\subsection{Intrinsic Evaluation}

Intrinsic evaluation methods measure the accuracy of a system in performing the task which it was designed to carry out. In the present case, it is the accuracy with which an entity can be classified as animate or inanimate. In order to assess the performance of the systems, four measures are considered:

$$
\begin{gathered}
\text { Accuracy }=\frac{\text { Correctly classified items }}{\text { Total } \text { number of items }} \\
\text { Precision }=\frac{\text { True positives }}{\text { True positives }+ \text { False positives }} \\
\text { Recall }=\frac{\text { True positives }}{\text { True positives }+ \text { False negatives }} \\
F-\text { measure }=\frac{2 * \text { Precision } * \text { Recall }}{\text { Precision }+ \text { Recall }}
\end{gathered}
$$




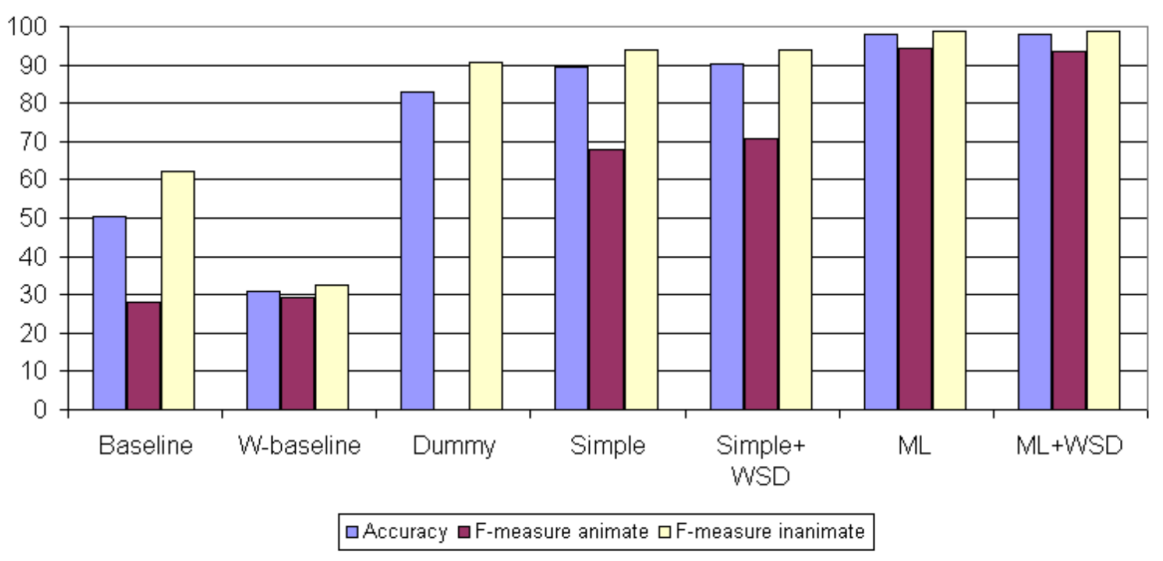

Figure 3: Evaluation of methods on AI corpus

The accuracy (1) measures how well a system can correctly classify a reference to an entity as animate or inanimate, but it can be misleading because of the large number of inanimate entities mentioned in texts. As is clear from Table 1, even though the texts were chosen so as to contain a large number of references to animate entities, the ratio between the number of references to animate entities and inanimate entities is approximatively 1 to 7.5 for SEMCOR, and 1 to 4.8 for AI. This means that a method which classifies all references to entities as inanimate would have an accuracy of $88.21 \%$ on SEMCOR and $82.77 \%$ on AI. As can be seen in Figures 3 and 4, as well as in Table 4, these results are not very far from the accuracy obtained by the system described in Section 4.1. However, as mentioned earlier, the intention is to use the filtering of references to animate entities for anaphora resolution and therefore, the use of a filter which classifies all the references as inanimate would be highly detrimental.

It is clearly important to know how well a system is able to identify references to animate and inanimate entities. In order to measure this, precision (2) and recall (3) are used for each class. The precision with which a system can identify animate references is defined as the ratio between the number of references correctly classified by the system as animate and the total number of references it classifies as animate (including the wrongly classified ones). A method's recall in classifying references to animate entities is defined as the ratio between the number of references correctly classified as animate and the total number of animate references to be classified. The precision and recall of inanimate classification is defined in a similar manner. The $f$-measure (4) combines precision and recall into one value. Several formulae for f-measure were proposed, the one used here gives equal importance to precision and recall.

Figures 3 and 4 , as well as Table 4 at the end of the paper, present the accuracy of the classification, and f-measures for classifying the animate and inanimate references. In addition to the methods presented in Section 4, three baseline methods were introduced. The first one classifies a reference to an entity as animate or inanimate on a random basis and is referred to in the figures as baseline. A second random baseline was introduced 


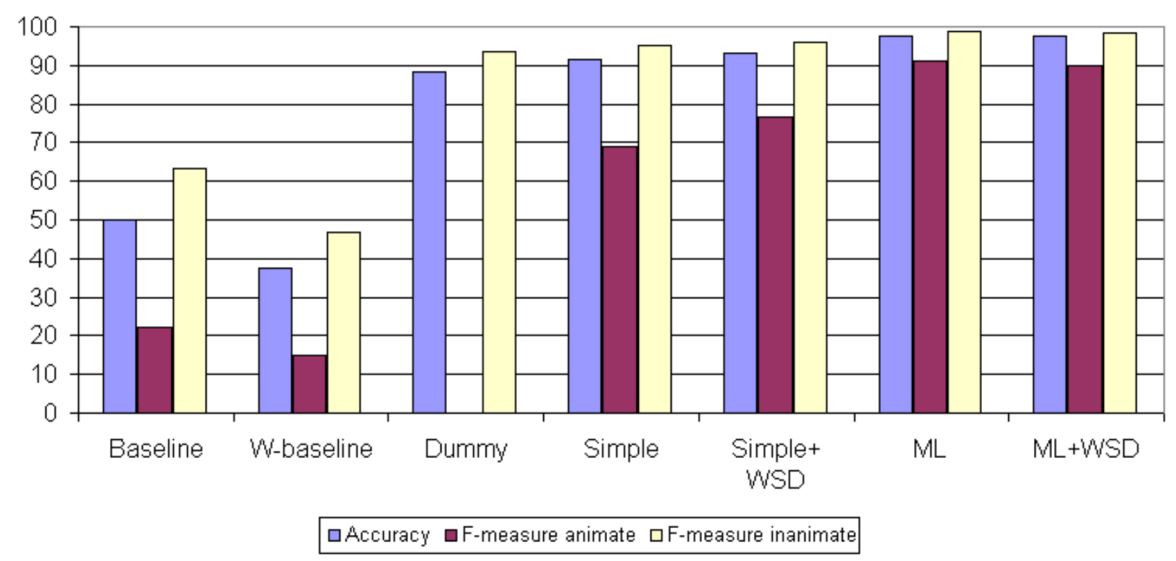

Figure 4: Evaluation of methods on SEMCOR corpus

because it was assumed that the number of gender marked pronouns in a text can indicate how likely it is that a particular noun appearing in that text will be animate or inanimate. In this case, the probability of a reference to be animate is proportional to the number of animate pronouns in the text and the classification is made on a weighted random basis. A similar rule applies for inanimate references. This second baseline is referred to in the figures as $W$-baseline. For purposes of comparison, a method was included which classifies all references as inanimate. This method is referred to as the dummy method.

Figures 3 and 4 show that all the other methods significantly outperform the baselines used. Close investigation of the figures, as well as of Table 4, shows that, for both corpora, the best method is the one which uses machine learning (presented in Section 4.2). It obtains high accuracy when classifying references to both animate and inanimate entities. In terms of accuracy, the simple method performs unexpectedly well, but it fails to accurately classify references to animate entities. Moreover, comparison with the dummy method on both files shows that the results of the simple method are not much better, which suggests that the simple method has a bias towards recognition of references to inanimate entities. The integration of word sense disambiguation yields mixed results: it increases the accuracy of the simple method, but it slightly decreases the performance of the machine learning method.

The relatively poor accuracy of the Simple system was expected and can be explained by the fact that the unique beginners, which are used as the basis for classification in that method, cover a range of senses which is too wide to be classified as belonging to a single animate or inanimate class. They are too general to be used as the basis for accurate classification. Additionally, the rules used to assist classification only provide limited recall in identifying animate references.

Comparison between the accuracy of the machine learning method and the level of interannotator agreement reveals that the automatic method agrees with the first annotator more than the second annotator does. As a result of this, it can be concluded that the accuracy of the automatic method matches human performance levels. 


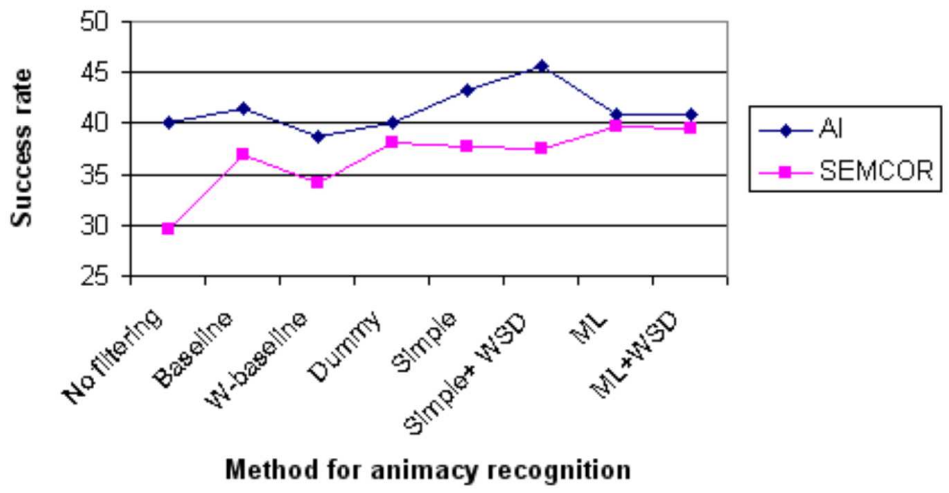

Figure 5: The accuracy of MARS when the different animacy filters are applied

\subsection{Extrinsic evaluation}

In the previous section, the performance of the classification methods was evaluated and it was demonstrated that even simple methods can achieve high accuracy at the expense of low precision and recall in the classification of references to animate entities. In computational linguistics, the output of one method is often used as the input for another one, and therefore it is important to know how the results of the first method influence the results of the second. This kind of evaluation is called extrinsic evaluation. Given that the identification of references to animate entities is not very useful in its own right, but can be vital for tasks like anaphora resolution, it is necessary to perform extrinsic evaluation too. In the case of this evaluation, the assumption is that the performance of anaphora resolution can be improved by filtering out candidates which do not agree in animacy with each referential pronoun.

The influence of animacy identification on anaphora resolution is thus evaluated using MARS (Mitkov, Evans, \& Orăsan, 2002), a robust anaphora resolver which relies on a set of boosting and impeding indicators to select an antecedent from a set of competing candidates. Due to the fact that the evaluation of MARS requires the manual annotation of pronouns' antecedents, which is a time consuming task, this evaluation was carried out only on a part of the corpus. To this end, the entire Amnesty International corpus as well as 22 files from the SEMCOR corpus have been used. Given that the animacy identifier can only influence the accuracy of anaphora resolvers with respect to third person singular pronouns, the accuracy of the resolver is reported only for these pronouns. Accuracy in anaphora resolution was calculated as the ratio between the number of pronouns correctly resolved and the total number of third person singular pronouns appearing in the test data. Figure 5 and Table 5 display this accuracy for alternate versions of MARS that exploit different methods for animacy identification.

MARs was designed to process texts from the technical domain, and therefore its performance is rather poor on this test corpus. Moreover, its performance can vary greatly from one domain to another. In light of the fact that the results of a different anaphora resolver may be very different on the same set of data, in addition to the performance of 

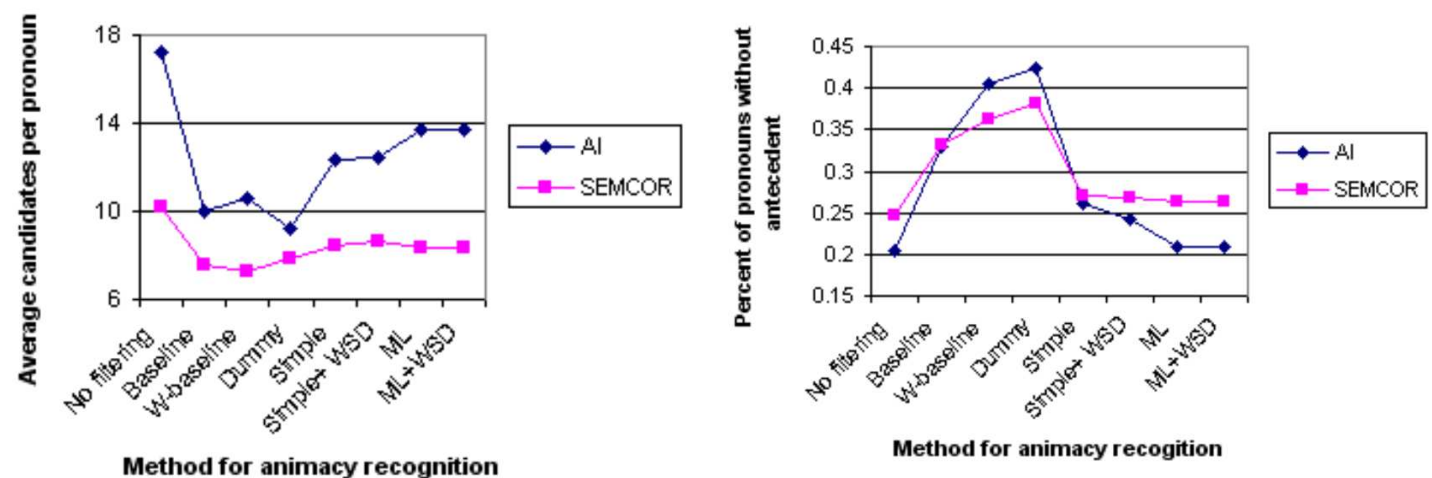

Figure 6: The average number of candidates and the percentage of pronouns without correct candidates when different animacy filters are applied

MARS with respect to third person singular pronouns, Figure 6 and Table 5 also present the reduction in the number of candidates that results from the animacy filtering, and the increase in the number of pronouns whose sets of competing candidates contain no valid antecedents as a result of this filtering. The former number is presented as the average number of candidates per pronoun, and the latter as the percentage of pronouns without valid antecedents in the list of candidates. The justification for reporting these two measures is that a good animacy filter will eliminate as many candidates as possible, but will not eliminate antecedents and leave pronouns without any correct candidates to be resolved to. ${ }^{9}$

As can be seen in Figure 5 and Table 5, regardless of which animacy identification method is used, the accuracy of the anaphora resolver improves. The degree of improvement varies from one corpus to another, but the pattern regarding the reduction in the number of candidates and the increase in the number of pronouns whose sets of competing candidates contain no valid antecedent is the same across both corpora. For the AI corpus, the best performance is obtained when the simple method enhanced with word sense disambiguation is used, followed by the simple method. Both improvements are statically significant ${ }^{10}$, as well as the difference between them. Both versions of the machine learning method improve the success rate of MARS by a small margin which is not statistically significant, but they increase the number of pronouns with no valid antecedent to select by only one, an increase which is not statistically significant. For the simple methods, the increase in the number of this type of pronoun is much larger and is statistically significant. Therefore in the case of the AI corpus, it can be concluded that, for MARS, a more aggressive method for filtering out candidates, such as the simple method with word sense disambiguation, is more appropriate. However, it is possible that for other anaphora resolution methods this result is not valid because they may be more strongly influenced by the increase in the number of pronouns with no valid antecedent to select.

9. It should be noted that, even without filtering, there are pronouns which do not have any candidates due to errors introduced by preprocessing tools such as the NP extractor which fails to identify some of the NPs.

10. In all the cases where we checked whether the differences between two results are statistically significant we used t-test with 0.05 confidence level. 
Processing the SEMCOR corpus, the best results for MARS are obtained by the machine learning method without the WSD module followed by the one which performs WSD. In both cases the increase over the performance of the unfiltered version is statistically significant, but the differences between the two machine learning methods are too small to be significant. In addition, these two methods ensure a large reduction in the number of candidates with the smallest increase in the number of pronouns whose sets of competing candidates contain no valid antecedent, an increase which is not significant.

As expected, the three baselines perform rather poorly. All three of them reduce the number of candidates at the expense of a high increase in the number of pronouns with no valid antecedent available for selection. Both the reduction in the number of candidates and the increase in the number of pronouns with no valid antecedent are statistically significant when compared to the system that does not use any filtering.

The results of MARS's performance are rather mixed when these baselines are used. For the AI corpus, the random baseline leads to a better result for MARS than the machine learning methods, but the differences are not statistically significant. However, this is achieved with a large increase in the number of pronouns which cannot be correctly resolved because all their valid antecedents have also been filtered by the method. For the AI corpus, application of the other two baselines led to results worse than or equal to those of MARS when no filtering is applied as a result of the large drop in the number of candidates.

For the SEMCOR corpus, all three baselines give rise to statistically significant improvements in performance levels over those obtained when no filtering is applied, but this is achieved by dramatically reducing the number of candidates considered. Integration of the dummy method into MARS leads to results which are better than the simple methods but, as argued before, this method is not appropriate for anaphora resolvers because it prevents them from correctly resolving any animate pronoun.

Investigation of the results revealed that for about $31 \%$ of the candidates it was not possible to apply any animacy filtering. There are three reasons for this. First, in the majority of cases, candidates are named entities which, as mentioned in Section 2, are not tackled by our method, though they constitute a relatively high proportion of the noun phrases occurring in the chosen texts. A second reason for these cases is the fact that some of the words are not present in WordNet and as a result, they are ignored by our method. Finally, in a limited number of cases the noun phrases identified by MARs did not match those identified by our animacy identifiers and for this reason it was not possible to classify them. ${ }^{11}$

\subsection{Extrinsic Evaluation on Simulated Data}

The results presented in the previous section makes it difficult to have a clear idea about how accurate the animacy identifier needs to be in order to have a significant positive influence on the performance of MARS. In light of this, we performed an experiment in which animacy identifiers which perform with a predefined accuracy were simulated. These systems were designed in such a way that the precision of animacy identification varies in $1 \%$ increments

11. The animacy identifiers proposed in this paper use both the NP and its context (i.e. the verb on which it depends and the number of pronouns in the text) and therefore they have to be run independently from any other module which uses their results. 

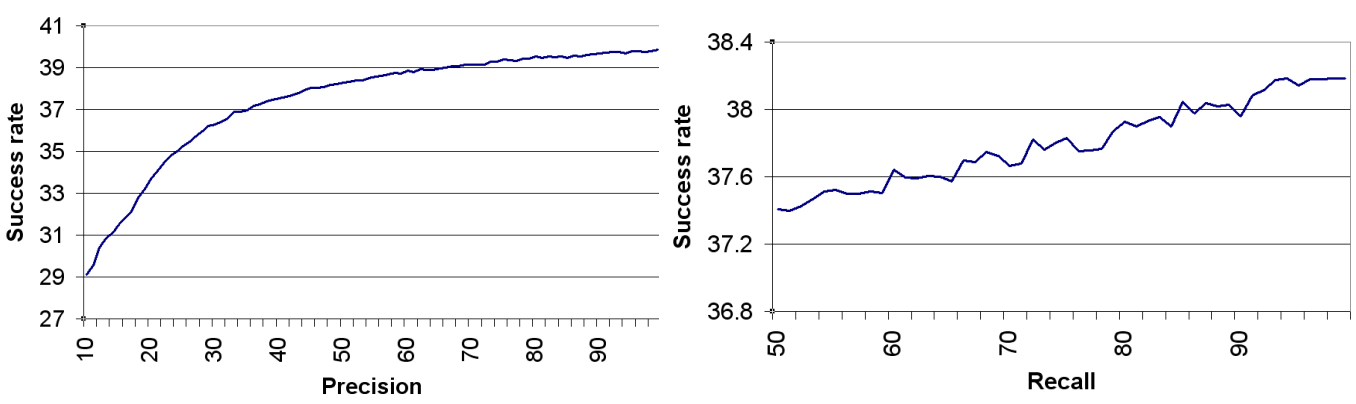

Figure 7: The evolution of success rate with changes in precision and recall

from $10 \%$ to $100 \%$, whilst recall varies from $50 \%$ to $100 \% .{ }^{12}$ In order to achieve this, we introduced a controlled number of errors in the annotated data by randomly changing the animacy of a predetermined number of noun phrases. In order to ensure fair results, the experiment was run 50 times for each precision-recall pair, so that a different set of entities were wrongly classified in each run. The list of classified entities (in this case derived directly from the annotated data and not processed by any of the methods described in this paper) was then used by MARS in the resolution process. Figure 7 presents the evolution of success rate as recall and precision are changed. In order to see how the success rate is influenced by the increase in recall, we calculated the success rates corresponding to the chosen recall value and all the values for precision between $10 \%$ and $100 \%$ and averaged them. In the same way we calculated the evolution of success rate with changes in precision.

As can be seen in the figures, precision has a greater influence on the success rate of MARS than recall because by increasing precision, we notice an almost continuous increase in the success rate. Overall, increasing recall also leads to an increase in the success rate, but this increase is not smooth. On the basis of this, we can conclude that high precision of animacy identification is more important than recall. These results are also supported by Table 5 where the Simple method leads to good performance for MARS despite its low recall (but higher precision) in the identification of animate entities.

Our experiments also reveal that for values higher than $80 \%$ for precision and recall, the success rate can vary considerably. For this reason we decided to focus on this region. Figure 8 presents the success rate corresponding to different precision-recall pairs using a contour chart. The darker areas correspond to higher values of success rate. As noticed before, the areas which correspond to high precision and high recall also feature high success rates, but it is difficult to identify clear thresholds for precision and recall which lead to improved performance especially because most of the differences between the first four intervals are not statistically significant.

12. We decided to control only the precision and recall of animacy identification because in this way, indirectly, we also control the recall and precision of the identification of inanimate entities. 


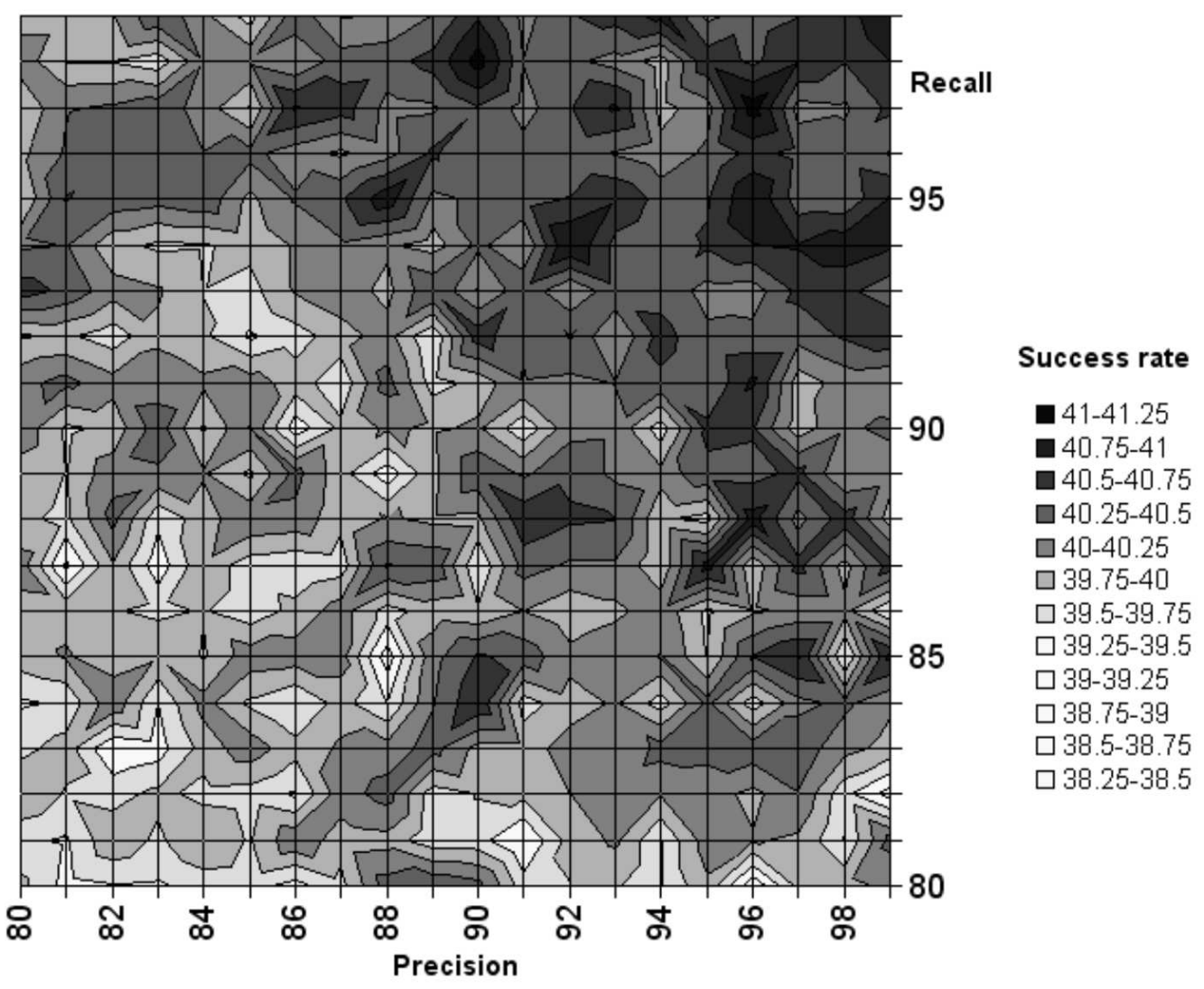

Figure 8: Contour chart showing the success rate for different values of precision and recall

\subsection{The Complexity of the Systems}

One aspect which needs to be considered whenever a system is developed is its complexity. This becomes a very important issue whenever such a system is integrated with a larger system, which needs to react promptly to its input (e.g. systems which are available over the Web). In the present case, each method presented in Section 4 is more complex than the previous one, and therefore requires more time to run. Table 3 shows the time necessary to run each system on the two corpora. As can be seen, the fastest method is the simple method which has a complexity proportional to $n^{*} m$ where $n$ is the number of entities in the entire corpus, and $m$ is the average number of senses for each word in WordNet. The method which uses machine learning is slower because it has to prepare the data for the machine learning algorithm, a process which has a similar complexity to the simple method, and in addition it has to run the memory-based learning algorithm, which compares each new instance with all instances already seen. Even though TimBL, the machine learning algorithm used, employs some sophisticated indexing techniques to speed up the process, for large training sets, the algorithm is slow. It has been noted that k-NN is an extremely slow classifier and the use of alternate ML algorithms, such as maximum entropy, may lead to quicker classification times with no loss in accuracy. When word sense disambiguation is 


\begin{tabular}{|c|c|c|}
\hline Method & AI & SEMCOR \\
\hline Simple method & 3 sec. & 25 sec. \\
\hline ML & 51 sec. & 286 sec. \\
\hline Simple+WSD & \multicolumn{2}{|c|}{ Several hours } \\
\hline ML+WSD & \multicolumn{2}{|c|}{ Several hours } \\
\hline
\end{tabular}

Table 3: The run time necessary for different methods

used, the processing time increases dramatically, because the complexity of the algorithm used is $n^{m}$ where $n$ is the number of distinct nouns from a text to be disambiguated, and $m$ is the average number of senses from WordNet for each noun. When the performance and run time of the methods is considered, the best performing method is ML, which ensures high accuracy together with relatively low execution time. The use of an alternate WSD method that exploits N-best lists, rather than considering all possible assignments of word senses, would be likely to improve the speed of disambiguation. An approach of this type has not yet been tested in our current work.

\section{Related Work}

With regard to work concerned with recognition of NP animacy, the sole concern in this section is with those methods which tackle the problem in English texts, a problem concerned with semantics that cannot be addressed using morphological information, as it can be in other languages.

Identification of the specific gender of proper names has been attempted by Hale and Charniak (1998). That method works by processing a 93931-word portion of the PennTreebank corpus with a pronoun resolution system and then noting the frequencies with which particular proper nouns are identified as the antecedents of feminine or masculine pronouns. Their paper reports an accuracy of $68.15 \%$ in assigning the correct gender to proper names.

The approach taken by Cardie and Wagstaff (1999) is similar to the simple statistical one described in Section 4.1, though the one described in this paper exploits a larger number of unique beginners in the ontology, considers semantic information about the verbs for which NPs are arguments, and also considers all possible senses for each noun. In the approach presented by Cardie and Wagstaff (1999), nouns with a sense subsumed by particular nodes in the WordNet ontology (namely the nodes human and animal) are considered animate. In terms of gender agreement, gazetteers are also used to assign each NP with a value for gender from the set of MASCuline, FEMinine, EITHER (which can be assumed to correspond to animate), or NEUTER. The method employed by Cardie and Wagstaff is fairly simple and is incorporated as just one feature in a vector used to classify coreference between NPs. The employed machine learning method blindly exploits the value assigned to the animacy feature, without interpreting it semantically. WordNet has been used to identify NP animacy in work by Denber (1998). Unfortunately, no evaluation of the task of animacy identification was reported in those papers. 


\section{Conclusions}

Animacy identification is a preprocessing step which can improve the performance of anaphora resolution in English by filtering out candidates which are not compatible, in terms of their agreement features, with the referential expression. In this paper, a more specific definition for animacy is used than the one proposed by Quirk et al. (1985). The adopted definition is more appropriate and conveys the usefulness of this feature in anaphora resolution. In the present study, the animacy of a noun phrase is determined by the fact that it can be referred to by means of a masculine or feminine pronoun as well as their possessive, relative and reflexive forms.

In this paper, two different animacy identifiers were presented and evaluated. The first one relies on the unique beginners from WordNet in combination with simple rules to determine the animacy of a noun phrase. Given that the unique beginners are too general to be used in this way and that the rules were designed through naïve observations, a second method was proposed. This second approach relies on a machine learning method and an enhanced WordNet to determine the animacy of a noun phrase. In addition to the normal semantic information, this enhanced WordNet contains information about the animacy of a synset. This animacy information was automatically calculated on the basis of manual annotation of the SEMCOR corpus with animacy information.

The two animacy identifiers were evaluated using intrinsic and extrinsic methods. The intrinsic evaluation employed several measures to determine the most appropriate identifier. Comparison between the results of these methods revealed that it is easy to obtain relatively high overall accuracy at the expense of low accuracy for the classification of animate references. For this reason, it was concluded that the extra resources required by the machine learning method, the best performing method, are fully justified. Inter-annotator agreement was measured in order to ascertain the difficulty of the task and as a result of this, it was noted that the machine learning method reaches a level of performance comparable to that of humans.

The extrinsic evaluation focused on how the performance of MARS, a robust anaphora resolver, is influenced by the animacy identifier. In light of the fact that MARS was designed to resolve anaphors in texts from a different genre, the results reported in the extrinsic evaluation did not focus only on the accuracy of that system, but also on how many candidates are removed by the animacy identifier and how many pronouns are left with no valid antecedent to select from their sets of candidates as a result of this process. Evaluation of MARS revealed that both of the methods proposed in this paper improve its accuracy, but the degree of improvement varies from one corpus to another. In terms of the reduction of the number of candidates that the anaphora resolution system has to consider, the machine learning method eliminates the fewest candidates, but as a result it only evokes small increases in the number of pronouns whose sets of competing candidates contain no valid antecedents. For this reason, we argue that extrinsic evaluation also shows that the machine learning approach is the most appropriate method to determine the animacy of noun phrases.

Experiments with WSD produced mixed results. Only on one of the corpora used in this research did it lead to small improvements in performance. We thus conclude that the extra computation required in order to disambiguate words is unnecessary. 


\begin{tabular}{|c|c|c|c|c|c|c|c|}
\hline \multirow{2}{*}{ Experiment } & \multirow{3}{*}{ Acc } & \multicolumn{3}{|c|}{ Animacy } & \multicolumn{3}{|c|}{ Inanimacy } \\
\cline { 5 - 8 } & & Prec & Recall & F-meas & Prec & Recall & F-meas \\
\hline \hline \multicolumn{7}{|c|}{ On AI corpus } \\
\hline Random baseline & $50.60 \%$ & $19.37 \%$ & $52.13 \%$ & $28.24 \%$ & $82.11 \%$ & $50.32 \%$ & $62.39 \%$ \\
\hline Weighted baseline & $31.01 \%$ & $18.07 \%$ & $76.48 \%$ & $29.23 \%$ & $79.27 \%$ & $20.60 \%$ & $32.70 \%$ \\
\hline Dummy method & $82.77 \%$ & $0 \%$ & - & - & $82.77 \%$ & $100 \%$ & $90.57 \%$ \\
\hline Simple system & $89.61 \%$ & $94.79 \%$ & $52.69 \%$ & $67.73 \%$ & $88.93 \%$ & $99.24 \%$ & $93.80 \%$ \\
\hline Simple system + WSD & $90.14 \%$ & $81.60 \%$ & $62.57 \%$ & $70.83 \%$ & $91.60 \%$ & $96.66 \%$ & $94.06 \%$ \\
\hline Machine learning system & $98.04 \%$ & $96.31 \%$ & $92.19 \%$ & $94.20 \%$ & $98.33 \%$ & $99.26 \%$ & $98.79 \%$ \\
\hline Machine learning with WSD & $97.85 \%$ & $95.37 \%$ & $92.00 \%$ & $93.65 \%$ & $98.34 \%$ & $99.07 \%$ & $98.70 \%$ \\
\hline \hline \multicolumn{7}{|c|}{ On SEMCOR corpus } \\
\hline Random baseline & $50.19 \%$ & $14.11 \%$ & $50.49 \%$ & $22.05 \%$ & $86.19 \%$ & $50.14 \%$ & $63.39 \%$ \\
\hline Weighted baseline & $37.62 \%$ & $8.40 \%$ & $74.44 \%$ & $15.09 \%$ & $88.41 \%$ & $31.64 \%$ & $46.60 \%$ \\
\hline Dummy method & $88.21 \%$ & $0 \%$ & - & - & $88.21 \%$ & $100 \%$ & $93.73 \%$ \\
\hline Simple system & $91.42 \%$ & $88.48 \%$ & $56.42 \%$ & $68.90 \%$ & $91.81 \%$ & $98.51 \%$ & $95.04 \%$ \\
\hline Simple system + WSD & $93.33 \%$ & $88.88 \%$ & $67.14 \%$ & $76.50 \%$ & $93.94 \%$ & $98.38 \%$ & $96.11 \%$ \\
\hline Machine learning system & $97.72 \%$ & $91.91 \%$ & $89.99 \%$ & $90.93 \%$ & $98.75 \%$ & $98.57 \%$ & $98.65 \%$ \\
\hline Machine learning with WSD & $97.51 \%$ & $89.97 \%$ & $90.14 \%$ & $90.05 \%$ & $98.59 \%$ & $98.56 \%$ & $98.57 \%$ \\
\hline
\end{tabular}

Table 4: The results of the classification 


\begin{tabular}{|c|c|c|c|}
\hline System & Average candidates per pronouns & Percentage of pronouns without antecedent & MARS accuracy \\
\hline \hline \multicolumn{2}{|c|}{ Results on the AI Corpus: 215 animate pronouns } \\
\hline No filtering & 17.20 & 20.46 & $40.00 \%$ \\
\hline Simple & 12.37 & 26.04 & $43.26 \%$ \\
\hline Simple + WSD & 12.47 & 24.18 & $45.58 \%$ \\
\hline Machine learning & 13.71 & 20.93 & $40.93 \%$ \\
\hline Machine learning + WSD & 13.70 & 20.93 & $40.93 \%$ \\
\hline Random baseline & 9.95 & 33.02 & $41.40 \%$ \\
\hline Weighted baseline & 10.57 & 40.46 & $38.60 \%$ \\
\hline Dummy method & 9.17 & 42.32 & $40.00 \%$ \\
\hline \hline Nesults on part of SEMCOR: 1250 animate pronouns & 24.80 \\
\hline No filtering & 10.20 & 26.96 & $29.60 \%$ \\
\hline Simple & 8.44 & 26.88 & $37.60 \%$ \\
\hline Simple + WSD & 8.66 & 26.32 & $37.50 \%$ \\
\hline Machine learning & 8.33 & 33.12 & $39.60 \%$ \\
\hline Machine learning + WSD & 8.33 & 36.16 & $39.52 \%$ \\
\hline Random baseline & 7.55 & 38.16 & $36.96 \%$ \\
\hline Weighted baseline & 7.28 & & $34.08 \%$ \\
\hline Dummy method & 7.83 & & $38.16 \%$ \\
\hline
\end{tabular}

Table 5: The results of extrinsic evaluation 


\section{References}

Barbu, C., Evans, R., \& Mitkov, R. (2002). A corpus based analysis of morphological disagreement in anaphora resolution. In Proceedings of Third International Conference on Language Resources and Evaluation (LREC2002), pp. 1995 - 1999 Las Palmas de Gran Canaria, Spain.

Barlow, M. (1998). Feature mismatches and anaphora resolution. In Proceedings of DAARC2, pp. 34 - 41 Lancaster, UK.

Brennan, S. E., Friedman, M. W., \& Pollard, C. J. (1987). A centering approach to pronouns. In Proceedings of the 25th Annual Metting of the ACL, pp. 155 - 162 Stanford, California.

Cardie, C., \& Wagstaff, K. (1999). Noun phrase coreference as clustering. In Proceedings of the 1999 Joint SIGDAT conference on Emphirical Methods in NLP and Very Large Corpora (ACL'99), pp. 82 - 89 University of Maryland, USA.

Chomsky, N. (1981). Lectures on Government and Binding. Dordrecht: Foris.

Cristea, D., Ide, N., Marcu, D., \& Tablan, V. (2000). An empirical investigation of the relation between discourse structure and co-reference. In Proceedings of the 18th International Conference on Computational Linguistics (COLING2000), pp. 208 214 Saarbrucken, Germany.

Daelemans, W., Zavrel, J., van der Sloot, K., \& van den Bosch, A. (2000). TiMBL: Tilburg memory based learner, version 3.0, reference guide, ilk technical report 00-01. Ilk 00-01, Tilburg University.

Denber, M. (1998). Automatic resolution of anaphora in English. Tech. rep., Eastman Kodak Co, Imaging Science Division.

Evans, R., \& Orăsan, C. (2000). Improving anaphora resolution by identifying animate entities in texts. In Proceedings of the Discourse Anaphora and Reference Resolution Conference (DAARC2000), pp. 154 - 162 Lancaster, UK.

Fellbaum, C. (Ed.). (1998). WordNet: An Electronic Lexical Database. The MIT Press.

Frances, W., \& Kucera, H. (1982). Frequency Analysis of English Usage. Houghton Mifflin, Boston.

Hale, J., \& Charniak, E. (1998). Getting useful gender statistics from English text. Tech. rep. CS-98-06, Brown University.

Hobbs, J. (1976). Pronoun resolution. Research report 76-1, City College, City University of New York.

Hobbs, J. (1978). Pronoun resolution. Lingua, 44, 339-352. 
Kennedy, C., \& Boguraev, B. (1996). Anaphora for everyone: pronominal anaphora resolution without a parser. In Proceedings of the 16th International Conference on Computational Linguistics (COLING'96), pp. 113 - 118 Copenhagen, Denmark.

Landes, S., Leacock, C., \& Tengi, R. I. (1998). Building semantic concordances. In Fellbaum (Fellbaum, 1998), pp. $199-216$.

Lappin, S., \& Leass, H. J. (1994). An algorithm for pronominal anaphora resolution. Computational Linguistics, 20(4), 535 - 562.

Mitchell, T. M. (1997). Machine learning. McGraw-Hill Series in Computer Science. McGraw-Hill.

Mitkov, R. (1998). Robust pronoun resolution with limited knowledge. In Proceedings of the 18th International Conference on Computational Linguistics (COLING'98/ACL'98), pp. 867 - 875 Montreal, Quebec, Canada.

Mitkov, R. (2002). Anaphora resolution. Longman.

Mitkov, R., Evans, R., \& Orăsan, C. (2002). A new, fully automatic version of Mitkov's knowledge-poor pronoun resolution method. In Proceedings of CICLing-2002, pp. 168 - 186 Mexico City, Mexico.

Ng, V., \& Cardie, C. (2002). Improving machine learning approaches to coreference resolution. In Proceedings of the 40th Annual Meeting of the Association for Computational Linguistics (ACL2002), pp. 104 - 111 Philadelphia, Pennsylvania.

Quinlan, J. R. (1993). C4.5: Programs for Machine Learning. Morgan Kaufmann.

Quirk, R., Greenbaum, S., Leech, G., \& Svartvik, J. (1985). A Comprehensive Grammar of the English Language. Longman.

Resnik, P. (1995). Disambiguating noun groupings with respect to Wordnet senses. In Yarovsky, D., \& Church, K. (Eds.), Proceedings of the Third Workshop on Very Large Corpora, pp. 54-68 Somerset, New Jersey. Association for Computational Linguistics.

Sirkin, R. M. (1995). Statistics for the social sciences. SAGE Publications.

Sparck Jones, K., \& Galliers, J. R. (1996). Evaluating natural language processing systems: an analysis and review. No. 1083 in Lecture Notes in Artificial Intelligence. Springer.

Tapanainen, P., \& Järvinen, T. (1997). A non-projective dependency parser. In Proceedings of the 5th Conference of Applied Natural Language Processing, pp. 64 - 71 Washington D.C., USA. 